\title{
Utility of Precision Medicine in the Management of Diabetes: Expert Opinion from an International Panel
}

Sanjay Kalra • A. K. Das · Sarita Bajaj · Gagan Priya · Sujoy Ghosh · R. N. Mehrotra · Sambit Das · Parag Shah • Vaishali Deshmukh · Debmalya Sanyal · Sruti Chandrasekaran · Deepak Khandelwal · Amaya Joshi · Tiny Nair · Fatimah Eliana · Hikmat Permana · M. D. Fariduddin · Pradeep Krishna Shrestha · Dina Shrestha · Shayaminda Kahandawa · Manilka Sumanathilaka · Ahamed Shaheed · Aly Ahmed Abdel Rahim · Abbas Orabi · Ahmed Al-ani · Wiam Hussein $\cdot$ Dinesh Kumar $\cdot$ Khalid Shaikh

Received: October 30, 2019 / Published online: January 8, 2020

(C) The Author(s) 2020

\section{ABSTRACT}

Aim: The primary objective of this review is to develop a practice-based expert group opinion on the role of precision medicine with a specific focus on sulfonylureas (SUs) in diabetes management.

Enhanced Digital Features To view enhanced digital features for this article go to https://doi.org/10.6084/ m9.figshare.11395266.

S. Kalra $(\bowtie)$

Department of Endocrinology, Bharti Hospital and BRIDE, Karnal, Haryana, India

e-mail: brideknl@gmail.com

A. K. Das

Department of Endocrinology and Medicine, Pondicherry Institute of Medical Sciences, Puducherry, India

\section{S. Bajaj}

Department of Endocrinology, MLN Medical

College, Allahabad, Uttar Pradesh, India

\section{G. Priya}

Department of Endocrinology, Fortis Hospital, Chandigarh, Punjab, India

S. Ghosh

Department of Endocrinology and Metabolism, Institute of Post-Graduate Medical Education and Research (IPGMER), Kolkata, West Bengal, India
Background: The clinical etiology, presentation and complications of diabetes vary from one patient to another, making the management of the disease challenging. The pre-eminent feature of diabetes mellitus (DM) are chronically elevated blood glucose concentrations; however, in clinical practice, the exclusion of autoimmunity, pregnancy, pancreatic disease or injury and rare genetic forms of diabetes is crucial. Within this framework, precision medicine provides unique insights into the risk factors and natural history of DM. Precision medicine goes beyond genomics and

\section{R. N. Mehrotra}

Department of Endocrinology, Apollo Hospitals, Jubilee Hills, Hyderabad, Telangana, India

\section{S. Das}

Department of Endocrinology, Apollo Hospitals, Bhubaneswar, Odisha, India

\section{P. Shah}

Department of Endocrinology and Diabetes, Gujarat Endocrine Centre, Ahmedabad, Gujarat, India

\section{Deshmukh}

Department of Endocrinology, Deshmukh Clinic and Research Centre, Pune, Maharashtra, India

D. Sanyal Department of Endocrinology, KPC Medical College, Kolkata, West Bengal, India 
encompasses patient-centered care, molecular technologies and data sharing. Precision medicine has evolved in the field of diabetology. It has helped improve the efficacy of SUs, a class of drugs, which have been effectively used in the management of diabetes mellitus for decades, and it has enabled the expansion of SUs use in diabetes patients with genetic mutations.

Review Results: After due discussions, the expert group analyzed studies that focused on the use of SUs in diabetes patients with genomic variations and rare mutations. The expert group opined that SUs are important glucose-lowering drugs and that precision medicine helps in improving the efficacy of SUs by matching them to those patients who will benefit most.

Conclusion: Precision medicine opens new vistas for the effective use of SUs in unexpected patient populations, such as those with genetic mutations.

Keywords: Genetic Monogenic diabetes; Precision medicine; Sulfonylureas

S. Chandrasekaran

Department of Endocrinology and Diabetes, Dr Rela Institute of Medical Science (RIMC), Chennai, Tamil Nadu, India

D. Khandelwal

Department of Endocrinology and Diabetes, Maharaja Agrasen Hospital, New Delhi, India

A. Joshi

Department of Endocrinology and Diabetes,

Bhaktivedanta Hospital and Research Institute,

Mumbai, Maharashtra, India

T. Nair

Department of Cardiology, PRS Hospital,

Trivandrum, Kerala, India

F. Eliana

Department of Internal Medicine, Faculty of

Medicine, YARSI University, Jakarta, Indonesia

H. Permana

Department of Internal Medicine, Faculty of

Medicine, Padjadjaran University, Bandung,

Indonesia

\section{Key Summary Points}

The primary objective of this review is to develop a practice-based expert group opinion on the role of precision medicine, with a specific focus on sulfonylureas (SUs) in diabetes management.

During a 2-day international meeting held at Delhi, India, experts reviewed the literature for available evidence and discussed the importance of precision medicine in the management of diabetes mellitus.

The key discussion points of the experts covered the role of pharmacogenetic studies in delivering precision medicine to patients with type 2 diabetes mellitus, the role of gene polymorphisms in the pharmacogenetics of SUs and the benefits of introducing SUs into the management of neonatal diabetes, and are summarized in the document.

Precision medicine opens new vistas for the effective use of SUs in unexpected patient populations, such as those with genetic mutations.

M. D. Fariduddin

Department of Endocrinology of Bangabandhu Sheikh, Mujib Medical University, Dhaka, Bangladesh

P. K. Shrestha

Department of Internal Medicine, Tribhuwan

University Teaching Hospital, Kathmandu, Nepal

D. Shrestha

Department of Endocrinology, Norvic International Hospital, Kathmandu, Nepal

S. Kahandawa

Department of Endocrinology, Teaching Hospital Karapitiya, Karapitiya, Galle, Sri Lanka

M. Sumanathilaka

Department of Endocrinology, National Hospital of Sri Lanka, Colombo, Sri Lanka

A. Shaheed

Department of Internal Medicine, Indira Gandhi

Memorial Hospital, Malé, Republic of Maldives 


\section{INTRODUCTION}

Precision medicine is defined as 'an emerging approach for disease treatment and prevention that considers individual variability in genes, environment, and lifestyle for each person'. Precision medicine promotes the customization of healthcare through more precise prevention strategies and treatment approaches compared to a one-size-fits-all approach wherein prevention and treatment strategies are developed for generalized usage. It facilitates a better understanding of how the interaction of environmental factors with genetic variations influences health [1].

Precision medicine differs from personalized medicine with regards to approach and the model of healthcare delivery. Patient-centered medicine refers to treatment approach that considers the genetic constitution of the patient with attention to their preferences, beliefs, attitudes, knowledge and social context, whereas precision medicine refers to a healthcare model that goes beyond genomics and depends on data, analytics and information. Personalized medicine links the patients and the providers, while the precision medicine ecosystem links patients, providers, clinical laboratories and researchers [2].

A. A. A. Rahim

Department of Diabetes and Metabolism,

Alexandria University, Alexandria, Egypt

A. Orabi

Department of Internal Medicine, Faculty of

Medicine, Zagazig University, Zagazig, Egypt

A. Al-ani

Department of Internal Medicine, Hamad General

Hospital, Doha, Qatar

W. Hussein

Department of Endocrinology and Diabetes, Royal

Hospital, Manama, Bahrain

D. Kumar

Department of Endocrinology, NMC Specialty

Hospital, Abu Dhabi, United Arab Emirates

K. Shaikh

Department of Diabetes, Faculty of Internal

Medicine, Royal Oman Police Hospital, Muscat, Oman
The clinical relevance of precision medicine in treatment armamentarium of diabetes mellitus (DM) is expanding. Recent advances in our understanding of the genomic architecture of diabetes have resulted in the development of precision medicine to personalize diabetes prevention and management. Monogenic forms of diabetes, such as maturity-onset diabetes of the young (MODY) and neonatal diabetes, have unique mutations and require unique treatment, and studies on these forms have paved the way for precision medicine in diabetes [3].

In this context, at an international meeting held in India, experts reviewed the literature for available evidence on significant advances in precision medicine in the management of DM and also discussed the emerging role of sulfonylureas (SUs) in the treatment of DM within the framework of precision medicine.

\section{METHODOLOGY}

During a 2-day international meeting held at Delhi, India, experts reviewed the literature for available evidence and discussed the importance of precision medicine in the management of DM. Experts discussed the place of SUs in the treatment armamentarium of precision medicine. The key discussion points of the experts covered the role of pharmacogenetic studies in delivering precision medicine to DM patients, the role of gene polymorphisms in the pharmacogenetics of SUs and the benefits of introducing SUs into the management of neonatal diabetes; these discussion points are summarized as recommendations in the section "Panel Statement" towards the end of this article.

During the meeting the primary focus of the panel members was discussion of the utility of precision medicine in the management of patients with different categories of DM, including type $1 \mathrm{DM}$ (T1DM), type $2 \mathrm{DM}$ (T2DM) and other forms. The panel members also emphasized the expanding role of precision medicine in T2DM management and the beneficial use of SUs in T2DM management.

In compliance with ethics and guidelines, this article is based on previously conducted studies and does not contain any studies with 
human participants or animals performed by any of the authors.

\section{RESULTS}

\section{Precision Medicine in Diabetes Mellitus}

Diabetes mellitus is a heterogeneous disease, and the spectrum of diabetes with regards to the underlying mechanisms has broadened in the past few decades. Clinical insights into several different overlapping mechanisms that lead to diabetes suggest that individuals might have features of different biological alterations and hybrid forms. The variety of different hybrid forms of DM, including latent autoimmune disease of adults (LADA) and ketosis-prone diabetes (KPD), is suggestive of biological alterations. In diabetes care, the scope of precision diabetes applies both to the more common forms of diabetes, such as T1DM and T2DM, and to the relatively rare subtypes of diabetes, such as monogenic diabetes [4].

Knowledge of an accepted classification of $\mathrm{DM}$ is prudent to a good understanding of the clinical application of precision medicine in diabetes care. With this in mind, according to the American Diabetes Association (ADA) Standards of Medical Care in Diabetes-2019 [4], $\mathrm{DM}$ can be classified into the following general categories:

1. Type $1 \mathrm{DM}$. This type of DM occurs due to autoimmune beta-cell destruction.

2. Type $2 \mathrm{DM}$. This type of DM occurs due to progressive loss of beta-cell insulin secretion.

3. Gestational DM. This type of DM refers to diabetes diagnosed during the second or third trimester of pregnancy that was not clearly overt diabetes prior to gestation.

4. Specific types of DM due to other causes, such as monogenic diabetes syndromes (e.g. neonatal diabetes and MODY); diseases of the exocrine pancreas (e.g. cystic fibrosis and pancreatitis); drug- or chemical-induced diabetes (such as with glucocorticoid use, in the treatment of human immunodeficiency virus/acquired immunodeficiency syndrome [HIV/AIDS] or after organ transplantation) [5].

Precision diabetes also finds practical applications in the context of continuous glucose monitoring. The clinical applications of precision medicine in diabetes care is summarized in Table 1 (adapted from [4]).

\section{Different Models for Understanding Diabetes Clusters}

Researchers have noted the heterogenous nature of diabetes and attempted to identify different variables that can help improve our understanding of the pathophysiological architecture of T2DM.

Table 1 Application of precision diabetes (adapted from [4])

Application of precision diabetes

Polygenic diabetes

T2DM

Prediction of development of diabetes

Response to therapy

Prediction of development of complications

Identifying clusters of T2DM

T1DM

Beta-cell function-completely absent/partially

preserved

Insulin autoantibodies_-present/absent

Risk of complications-low/high

Monogenic diabetes

Maturity-onset diabetes of the young (MODY)

Neonatal diabetes (onset of diabetes at $<6$ months of age)

Congenital hyperinsulinemic hypoglycemia

Monitoring and follow-up

TIDM Type 1 diabetes mellitus, T2DM type 2 diabetes mellitus 
Ahlqvist et al. used patient data from Swedish patients who had beene recently diagnosed with diabetes. During the study, machine learning algorithms were applied, and diabetes subtypes were classified. The investigators identified five subtypes based on six different variables (age at diagnosis, body mass index, glycated hemoglobin, glutamate decarboxylase antibodies, homeostasis model assessment, and c-peptide) [6].

McCarthy et al. proposed a different model, called the 'palette model,' which categorizes every individual according to the various pathophysiological processes that have been identified to contribute to the risk and progression of diabetes. The model considers that every person with diabetes will have multiple parallel defects that will affect several other processes [7].

Although it is well accepted that the role of precision medicine in diabetes management is indisputable, the path by which prescision medicine can be used to optimize the prevention and treatment of diabetes and other diseases is still unclear. In this regard, a few precision medicine initiatives have been proposed that present a clear plan for clinical translation. Some of the factors that should be considered in order to achieve this objective include an understanding of the disease, the identification of disease-specific biomarkers, vertical integration of biomarkers and algorithms, regulatory agencies and approval, patient engagement, testing of biomarker/s in clinical trials, clinician engagement, regulatory approval, implications on the economics of healthcare, clinical translation (including continuing education of clinicians and support) and patient feedback [8].

\section{Global Initiatives on Precision Medicine Relevant to Type 2 Diabetes Mellitus}

The rapid growth of large data sets, machine learning and collaborative networks have given rise to many initiatives, many of which were started globally, with the aim to understand the role of precision medicines in T2DM.
A joint statement published by the ADA and European Association for the Study of Diabetes (EASD) contained a general recommendation on moving toward a 'patient-centered approach' from a protocol-driven approach. This position statement is based on an evaluation of the safety, efficacy and tolerability of each class of diabetes drugs and recommends that "Choice is based on patient preferences as well as various patient, disease, and drug characteristics, with the goal being to reduce glucose concentrations while minimizing side effects, especially hypoglycemia" [9].

In this regard, countries like Australia, China, the European Union, Sweden, Saudi Arabia, Nordic nations and the UK are not far behind [8].

\section{Role of Pharmacogenetics Studies in Delivering Precision Medicine to Patients with Diabetes Mellitus}

Pharmacogenetics is a branch of pharmacology that studies the modulation of therapeutic responses as a result of genetic variation and drug exposure. The feasibility of conducting pharmacogenetic studies has increased in recent years due to growing amount of data on human genetic variation and technological advancement. It is important to clarify the objectives of such studies, which are presented as follows [10]:

- Patient stratification: Can help identify and define subgroups of individuals who are likely to respond to clinical treatment or would experience side effects due to the drug.

- Target identification: Can ensure discovery of novel drugs that can illustrate mechanism of action and open new therapeutic avenues.

- Functional characterization: Will provide insights on the functional characteristics (mode of action) of the gene product or the cell type in which it is expressed, which will eventually help target the right medication to the right person and at the right time.

Metformin has been studied extensively to identify the genetic variants that reduce its 
efficacy. It was observed that genetic variants in the SLC22A1 (solute carrier family 22 member 1) gene encoding for organic cation transporter (OCT1), in the metformin transporter MATE1 (multidrug and toxin extrusion 1) gene, and in the $S L C 2 A 2$ (solute carrier family 2 member 2 ) gene encoding for sodium glucose transporter 2 (SGLT2) can alter metformin response [11].

\section{Role of Artificial Intelligence and Technological Advancements in Precision Medicine in Type 2 Diabetes Mellitus}

The development of precision medicine for diabetes is primarily based on technological advances, such as high-resolution "omics" assays, wearable devices that track the behavior of and exposure to drugs and digital imaging [8]. The use of "omics" technologies and large sample sizes have generated numerous huge data sets, and the analyses of these data sets require advanced computational and statistical methods. The big data revolution has provided an opportunity to apply artificial intelligence (AI) and machine learning algorithms to this vast amount of data. AI, machine learning algorithms, computational biology, and digital biomarkers provide the means to translate new data into required clinical information [12]. In addition, the intelligent integration of the data received from these technologies can provide detailed information on a person's physical condition, medical history, susceptibility to certain risk factors; predict the response to specific antidiabetic therapies; and track disease progression. Eventually, such information will not only help in the diagnosis but also in the prevention and management of T2DM [8].

\section{Ethical, Legal and Social Implications of Precision Medicine}

The role of precision medicine is expanding in several clinical areas and, concurrently, its ethical, legal and social implications are increasing in scope and complexity. Two broad issues of clinical importance can be identified that pertain to the ethical and social implications of precision medicine. The first refers to the consequences of the markedly increased amounts of health information associated with precision medicine. Current concerns related to such increased amounts of personal health information include the privacy of patient health information, discrimination, the physician-patient relationship and liability. The second issue refers to the potential of precision medicine to exacerbate disparities in healthcare and includes cost and access to healthcare and access to information technology.

A collaborative and proactive approach is crucial to address the ethical and social issues of precision medicine related to patient privacy, the physician-patient relationship and healthcare disparities [13].

\section{Place of Sulfonylureas in the Precision Medicine Armamentarium}

Although SUs are recommended as adjunctive therapy to lifestyle modification and metformin, the effect of this class of medications on cardiovascular outcomes remains controversial [14]. Emdin et al. reported that genetic variation can predict the risk of chronic heart disease (CHD) in patients on SU therapy [13]. In their study, the authors collected individual data from 120,286 participants of European ancestry from the UK biobank. As the target genes, they selected the ABCC 8 (ATP binding cassette subfamily $\mathrm{C}$ member 8) gene and the KCNJ11 (potassium voltage-gated channel subfamily $\mathrm{J}$ member 11) gene as SU initiates insulin release by binding to this complex. The ABCC 8 polymorphism A1369S mimics the SU effect in patients with T2DM, and hence the study also examined whether this gene was associated with CHD, stroke, heart failure, among others. The investigators found that variant p.A1369S was associated with a significantly lower risk of clinical consequence, such as CHD (odds ratio 0.98; 95\% confidence interval 0.96, 0.99; $P=5.9 \times 10^{-4}$ ), and with a higher BMI but lower waist-to-hip ratio. The results of this study therefore suggest that genetic variation that mimics SU effect is associated with reduced risk of T2DM and cardiovascular disease [15]. 


\section{Gene Polymorphisms in Pharmacogenetics of Sulfonylureas}

Evidence suggests that single nucleotide polymorphisms of the genes encoding the KATP channel have been related to the efficacy of secretagogue drugs (Table 2). Loss-of-function mutations of these genes constitute the predominant cause of congenital hyperinsulinism. Nearly 24 mutations have been characterized in the pancreatic beta-cell KATP channel subunit Kir6.2 (KCNJ11) and 150 mutations have been characterized in sulfonylurea receptor 1 (SUR1) protein $(A B C C 8)$ in patients with DM. Gain-offunction mutations in Kir6.2 or SUR1 cause neonatal diabetes. Neary 40 different neonatal diabetes mutations have been characterized in Kir6.2 and in SUR1. All Kir6.2 mutations cause dominant neonatal diabetes by reducing the ability of ATP to block the $\mathrm{K}_{\mathrm{ATP}}$ channel, thereby hyperpolarizing the $\beta$-cells and preventing insulin secretion. Consequently, the mutated $\mathrm{K}_{\mathrm{ATP}}$ channel does not close in response to increased ATP concentrations [16].

\section{Gene Variant-Related Pharmacokinetics of Sulfonylureas and Repaglinides}

The metabolism of SUs in the liver is primarily carried out by cytochrome P450 isoenzyme 2C9 encoded by CYP2C9. The major allele of this gene is $C Y P 2 C 9^{*} 1$. Evidence from clinical studies on healthy subjects have shown that the variant alleles are associated with increased plasma concentration and decreased clearance of SUs after oral administration.

Studies have shown that genetic variants that influence the pharmacokinetics of repaglinides are different from those of SUs. The pharmacokinetics of replaglinide is influenced by variants in $C Y P 2 C 8$ and variants in $521 \mathrm{~T}>\mathrm{C}$ in $S L C O 1 B 1$, the gene encoding organic anionic transporter B1 (OATPB1) [22].

Table 2 Summary of gene polymorphisms involved in the pharmacogenetics of sulfonylureas

\begin{tabular}{|c|c|c|}
\hline $\begin{array}{l}\text { Single } \\
\text { nucleotide } \\
\text { polymorphism }\end{array}$ & Study population & Associated response phenotype \\
\hline KCNJII [17] & $\begin{array}{l}\text { Patients with T2DM }(N=101) \text { treated with } \\
\text { gliclazide for } 6 \text { months }\end{array}$ & $\begin{array}{l}\text { K-allele carriers had significantly higher decrease in } \\
\mathrm{HbA}_{1 \mathrm{c}} \text { compared with EE homozygotes }\end{array}$ \\
\hline$A B C C 8[18]$ & Patients with T2DM $(N=251)$ on SU therapy. & $\begin{array}{l}\text { Patients with GG genotype had significantly higher } \\
\text { triglyceride levels compared to the patients with } \\
\text { AA genotype }\end{array}$ \\
\hline$K C N Q 1[19]$ & $\begin{array}{l}\text { T2DM patients }(N=87) \text { who failed to achieve } \\
\text { glycemic control on metformin therapy, treated } \\
6 \text { months with } \mathrm{SU}\end{array}$ & $\begin{array}{l}\text { Carriers of the T-allele (TT }+\mathrm{TG}) \text { achieved } \\
\text { significantly lower FPG levels compared to the } \\
\text { patients with the risk GG genotype }\end{array}$ \\
\hline TCF7L2 [20] & $\begin{array}{l}\text { T2DM patients }(N=189), 6 \text {-month SU } \\
\text { treatment }\end{array}$ & $\begin{array}{l}\mathrm{T} \text { allele was significantly more frequent in patients } \\
\text { who failed to respond to SU than in the control } \\
\text { subjects }\end{array}$ \\
\hline CYP2C9 [21] & $\begin{array}{l}\text { Population-based GoDARTS study: T2D patients, } \\
\text { incident users of SU }(N=1.073)\end{array}$ & $\begin{array}{l}\text { More likely to achieve a treatment } \mathrm{HbAlc}<7 \% \\
(53 \mathrm{mmol} / \mathrm{mol}) \text { than patients with the wild-type } \\
\text { genotype }\end{array}$ \\
\hline
\end{tabular}

$H b A_{1 c}$ Glycated hemoglobin, $S U$ sulfonylurea, $F P G$ fasting plasma glucose 


\section{Sulfonylureas for Treatment of Neonatal Diabetes}

Neonatal diabetes, as the name suggests, is diagnosed during infancy and at a frequency of 1 in 400,000 live births. In people younger than 20 years, roughly half (approx. 1:252,000) are diagnosed with neonatal diagnosis. Studies have indicated that up to $70 \%$ of such patients have mutations in the KCNJ11 and ABCC 8 genes [8]. Treatment with SUs has been reported to enable the successful discontinuation of insulin therapy in $90 \%$ patients diagnosed with neonatal diabetes [23]. In this regard, SUs can play a pivotal role in the management of neonatal diabetes [10]:

- It may be feasible to overcome the genetic defect through the administration of high doses of SUs that target the SU receptor/ potassium channel complex.

- It may be feasible to transition patients from exogenous insulin to oral regimen.

- Improved glycemic response ensures better quality of care and better quality of life.

Genetic testing in neonatal diabetes would result in high quality-of-life benefits at 10 years (0.32 quality-adjusted life-years; USD 12,528 saved) [8].

\section{Sulfonylureas for Monogenic Diabetes, a Rare Genetic Mutation}

In 2006, University of Chicago Medical Center physicians spotted an unusual genetic mutation in 6-year-old Lilly Jaffe-a finding that meant the girl could switch from painful insulin injections to pills as a means of controlling her T1DM.

Lilly was diagnosed with juvenile diabetes (T1DM), the more serious form, at 1 month of age. In June 2006, her parents learned from Dr. Louis Philipson, medical director of the Kovler Diabetes Center at the University of Chicago, about cutting-edge diabetes research that had been conducted in the UK. He told them that some children can undergo an unusual mutation of T1DM in their first 6 months of life but that their diabetes can be managed with pills and they do not need to take insulin injections.
Lilly was found to have the rare genetic mutation known as monogenic diabetes. A couple of months later, after treatment, Lilly was completely insulin free and taking five glyburide pills twice a day. Lilly's story was published in the newspaper (September 2006), which initiated hundreds of queries from families across the USA who wanted to know if their child might have a similar genetic mutation. Among the 70 patients who subsequently underwent genetic testing, some were found to have the same mutation as Lilly, but others had mutations in other genes. This initiated further research and the results were published in 2007.

In order to identify more patients and create more entries in the database of the study, University of Chicago doctors proposed the creation of the first state-mandated diabetes registry. The bill was officially signed by Gov. Pat Quinn in August 2009 and became Lilly's Law. The law necessitates Illinois physicians to register all children with diabetes onset before 12 months of age with the state Department of Public Health. This measure helps add more patients to the registry, which was approved as a 3 -year pilot program for further advancements [24].

\section{Sulfonylureas for MODY1 and MODY2}

There are 11 subtypes of MODY, based on the different mutations in genes that are responsible for insulin secretion or blood glucose control. MODY3 is caused by mutations in the hepatocyte nuclear factor-1alpha gene (HNF1A). It should be noted that MODY is often misdiagnosed as T1DM or T2DM due to overlapping signs, symptoms and risk factors [25].

- MODY1, caused by mutation in the HNF4A gene, can be managed by SUs; however, patients may eventually require insulin [25].

- MODY3 can be managed by switching to SUs, which corrects the genetic defect, and and insulin injections may then not be needed [25].

Patients with MODY1 and MODY3 should switching from insulin to SUs for better safety and quality of life. 


\section{Precision Medicine Aids in Predicting} Side-Effects of SUs

\section{Association Between CYP2C9*3 Allele and Risk of Hypoglycemia}

Sulfonylureas are inactivated by the liver microsomal CYP2C9 enzyme. However, around $6 \%$ of people carry a polymorphism ( ${ }^{*} 2$ and ${ }^{*} 3$ ) for reduced function in the gene for this enzyme. As a result, patients with T2DM carrying this polymorphism may be at a high risk of hypoglycemia as SUs are poorly inactivated in such individuals [23].

Ragia et al. conducted a study aimed at determining whether patients with T2DM with the CYP2C9 variant alleles CYP2C9*2 and $C Y P 2 C 9 * 3$ were at a high risk of hypoglycemic events when receiving the SUs glimepiride and gliclazide. The study population comprised 92 patients with T2DM who were receiving SU treatment and reporting drug-induced hypoglycemia and 84 patients with T2DM who were receiving SU treatment and having never experienced hypoglycemia. Genotype determination and logistic regression were performed. The investigators found that 11 of patients $(12 \%)$ experiencing hypoglycemia carried the CYP2C9*3 allele, in comparison to only one of 84 patients $(1.2 \%)$ who were free of SU-induced hypoglycemia [26]. The authors concluded that patients with T2DM carrying CYP2C $9 * 3$ are at a high risk of developing hypoglycemia when on treatment with SUs. One possible cause of hypoglycemic episodes in these patients is impaired metabolism that reduces the efficacy of antidiabetic drugs. Hence, CYP2C9 might be considered to be a useful tool for predicting the consequences of SU treatment in patients with DM [26].

\section{Interaction Between CYP2C9 and POR Genes Acts as an Important Determinant of Efficacy and Severe Adverse Effects of Sulfonylurea Treatment}

A study similar to that of Ragia et al. [26] was conducted by Dujic et al. to explore whether variants in the cytochrome $\mathrm{P} 450$ oxidoreductase (POR) gene can affect CYP2C9 activity and thus the efficacy of SU in patients with T2DM [27]. The study enrolled 1770 patients with T2DM for the analysis. A stratified analysis was performed, and the results indicated that reduced functions of CYP2C9 alleles were observed in patients with $P O R^{*} 1 /{ }^{*} 1$, which resulted in patients being susceptible to hypoglycemic episodes. In contrast, patients who carried $P O R{ }^{*} 28$ showed a better response to $\mathrm{SU}$ treatment (Table 3) [27].

The study results emphasize that the interaction between CYP2C9 and POR genes should be considered in order to predict the efficacy of SU treatment or the risk of adverse effects in patients with T2DM [27].

\section{PANEL STATEMENT}

Considering the available information, a panel of experts considered various ways to introduce precision medicine into clinical practice. The expert group recommended that the potential of precision medicine should be tapped for managing diabetes.

1. Precision medicine is a relatively new term, but the concept has been a part of healthcare for many years; for example, blood transfusion can be considered to be a part of precision medicine.

2. The ADA has established the 'Precision Medicine in Diabetes Task Force' to formulate a consensus statement on precision diabetes medicine as well as initiate complementary activities.

3. Pharmacogenetic studies can be helpful in achieving one or several major objectives, including patient stratification, target identification and functional characterization.

4. The drive to develop precision medicine for use in diabetes prevention and management is based on major technological advances, such as high-resolution "omics" assays, wearable devices that track the behaviors and exposures and digital imaging.

5. A collaborative and proactive approach is crucial to address the social and ethical issues of precision medicine related to 
Table 3 Effect of cytochrome P450 oxidoreductase gene status combined with the cytochrome P450 2C9 gene on hypoglycemia

\begin{tabular}{|c|c|c|c|}
\hline$P O R$ and/or $C Y P 2 C 9$ genotypes & Cases/control & Odds ratio $(95 \%$ confidence interval) & $P$ \\
\hline \multicolumn{4}{|c|}{ Effect of $C Y P 2 C 9$ reduced function polymorphism on hypoglycemia-analysis by $P O R$ genotype } \\
\hline \multicolumn{4}{|l|}{$P O R$ genotype } \\
\hline$P O R * R^{*} 1$ & $29 / 151$ & $2.81(1.30-6.09)$ & 0.009 \\
\hline POR * $1 / * 28, * 28 / * 28$ & $33 / 133$ & $0.70(0.32-1.57)$ & 0.390 \\
\hline \multicolumn{4}{|c|}{ Effect of $P O R^{*} 28$ on hypoglycemia-analysis stratified by $C Y P 2 C 9$ genotype } \\
\hline \multicolumn{4}{|l|}{ CYP2C9 genotype } \\
\hline CYP $2 C 9^{*} 1 /{ }^{*} 1$ & $35 / 180$ & $1.58(0.82-3.03)$ & 0.174 \\
\hline CYP $2 C 9{ }^{*} 2$ or ${ }^{*} 3$ carriers & $27 / 104$ & $0.58(0.26-1.28)$ & 0.177 \\
\hline
\end{tabular}

CYP2C9 Cytochrome P450 2C9, POR cytochrome P450 oxidoreductase

patient privacy, physician-patient relationship and health disparities

6. Patients need to be a part of precision medicine for it to achieve its potential to the fullest; therefore, patient rights and concerns must be a priority for policymakers.

7. Sulfonylureas are the classic precision medicines in the armamentarium to manage diabetes.

8. Neonatal diabetes is diagnosed in infancy and affects about 1:400,000 live births, and roughly half (approx. 1:252,000 in people aged $<20$ years) develop permanent neonatal diabetes. Treatment with sulfonylureas rectifies genetic defect in approximately $90 \%$ cases, allowing transition to an oral regimen and discontinuation of exogenous insulin.

9. CYP2C9 genotyping might be a useful tool for predicting adverse effects caused by sulfonylureas.

\section{CONCLUSION}

In coming years, the management of diabetes is likely to experience a dramatic change with the advent of precision medicine. However, understanding the molecular variations underlying different diabetes subtypes and integration and application of these data into daily clinical practice will require efforts from all components of healthcare. The use of big data, genomics and other "omics," such as metabolomics and transcriptomics, could make the dream of precision medicine in diabetes care become a reality in the near future. Rapid strides have been made in the management of monogenic forms of diabetes, and precision diabetes has gradually emerged as an important part of the clinical tools used at advanced diabetes centers. Precision medicine has paved the way for the effective use of SUs in certain patient populations with rare genetic mutations. In the coming years, rapid advances can be expected in the field of precision diabetes, thereby making the control of diabetes more effective and hopefully leading to prevention of its complications and improvement of the quality of life of people afflicted with diabetes.

\section{ACKNOWLEDGEMENTS}

All authors had full access to the articles reviewed in this manuscript and take complete responsibility for the integrity and accuracy of this manuscript. The content published herein solely represents the views and opinions of the authors. The details published herein are intended for informational, educational, academic and/or research purposes and are not intended to substitute for professional medical 
advice, diagnosis or treatment. Sanofi India helped in organization and logistic support for this expert forum meeting.

Funding. This expert opinion initiative has been supported by Sanofi India. The study sponsor is also funding the journal's Rapid Service and Open Access Fees.

Medical Writing and Editorial Assistance. Medical writing and editorial support was provided by Dr Rajshri Mallabadi and Dr Kavitha Ganesha from BioQuest Solutions Pvt. Ltd. which was paid for by Sanofi, India.

Authorship. All named authors meet the International Committee of Medical Journal Editors (ICMJE) criteria for authorship for this article, take responsibility for the integrity of the work as a whole, and have given their approval for this version to be published.

Disclosures. Sanjay Kalra is a member of the journal's Editorial Board. Das AK, Priya G, Ghosh S, Mehrotra RN, Das S, Shah P, Bajaj S, Deshmukh V, Sanyal D, Chandrasekaran S, Khandelwal D, Joshi A, Nair T, Eliana F, Permana H, Fariduddin MD, Shrestha PK, Shrestha D, Kahandawa S, Sumanathilaka M, Shaheed A, Niyaz M, Rahim AA, Orabi A, Al-ani A, Hussein W, Kumar D and Shaikh $\mathrm{K}$ have nothing to disclose.

Compliance with Ethics Guidelines. This article is based on previously conducted studies and does not contain any studies with human participants or animals performed by any of the authors.

Data Availability. Data sharing is not applicable to this article as no datasets were generated or analyzed during the current study.

Open Access. This article is distributed under the terms of the Creative Commons Attribution-NonCommercial 4.0 International License (http://creativecommons.org/licenses/ by-nc/4.0/), which permits any non commercial use, distribution, and reproduction in any medium, provided you give appropriate credit to the original author(s) and the source, provide a link to the Creative Commons license, and indicate if changes were made.

\section{REFERENCES}

1. Genetic Science Learning Center. What is Precision Medicine? Learn. Genetics. https://learn.genetics. utah.edu/content/precision/intro/. Accessed 11 Oct 2019.

2. Ginsburg GS, Phillips KA. Precision medicine: from science to value. Health Aff (Millwood). 2018;37(5): 694-701.

3. Prasad RB, Groop L. Precision medicine in type 2 diabetes. J Intern Med. 2019;285(1):40-8.

4. Merino J, Florez JC. Precision medicine in diabetes: an opportunity for clinical translation. Ann N Y Acad Sci. 2018;1411(1):140-152.

5. American Diabetes Association. 2. Classification and diagnosis of diabetes: standards of medical care in diabetes-2019. Diabetes Care. 2019;42[Suppl 1]: S13-S28.

6. Ahlqvist E, Storm $P$, Käräjämäki $A$, et al. Novel subgroups of adult-onset diabetes and their association with outcomes: a data-driven cluster analysis of six variables. Lancet Diabetes Endocrinol. 2018;6(5):361-9.

7. McCarthy MI. Painting a new picture of personalised medicine for diabetes. Diabetologia. 2017;60(5):793-9.

8. Fitipaldi H, McCarthy MI, Florez JC, et al. A global overview of precision medicine in type 2 diabetes. Diabetes. 2018;67(10):1911-22.

9. Inzucchi SE, Bergenstal RM, Buse JB, et al. Management of hyperglycemia in type 2 diabetes, 2015-a patient-centered approach: update to a position statement of the American Diabetes Association and the European Association for the Study of Diabetes. Diabetes Care. 2015;38(1):140-9.

10. Florez JC. Pharmacogenetics in type 2 diabetes: precision medicine or discovery tool? Diabetologia. 2017;60(5):800-7.

11. Xie F, Chan JC, Ma RC. Precision medicine in diabetes prevention, classification and management. J Diabetes Investig. 2018;9(5):998-1015.

12. Seyhan AA, Carini C. Are innovation and new technologies in precision medicine paving a new 
era in patients centric care? J Transl Med. 2019;17(1):114.

13. Brothers KB, Rothstein MA. Ethical, legal and social implications of incorporating personalized medicine into healthcare. Per Med. 2015;12(1):43-51.

14. Li Y, Hu Y, Ley SH, et al. Sulfonylurea use and incident cardiovascular disease among patients with type 2 diabetes: prospective cohort study among women. Diabetes Care. 2014;37(11): 3106-13.

15. Emdin CA, Klarin D, Natarajan P, et al. Genetic variation at the sulfonylurea receptor, type 2 diabetes, and coronary heart disease. Diabetes. 2017;66(8):2310-5.

16. Semiz S, Dujic T, Causevic A. Pharmacogenetics and personalized treatment of type 2 diabetes. Biochem Med (Zagreb). 2013;23(2):154-171.

17. Javorsky M, Klimcakova L, Schroner Z, et al. KCNJ11 gene E23K variant and therapeutic response to sulfonylureas. Eur J Intern Med. 2012;23(3):245-9.

18. Nikolac N, Simundic AM, Saracevic A, et al. ABCC8 polymorphisms are associated with triglyceride concentration in type 2 diabetics on sulfonylurea therapy. Genet Test Mol Biomarkers. 2012;16: 924-30.

19. Schroner Z, Javorsky M, Tkacova R, et al. Effect of sulphonylurea treatment on glycaemic control is related to TCF7L2 genotype in patients with type 2 diabetes. Diabetes Obes Metab. 2011;13:89-91.
20. Holstein A, Hahn M, Korner A, et al. TCF7L2 and therapeutic response to sulfonylureas in patients with type 2 diabetes. BMC Med Genet. 2011;12:3022.

21. Zhou K, Donnelly LA, Kimber $\mathrm{CH}$, et al. Reducedfunction SLC22A1 polymorphisms encoding organic cation transporter 1 and glycemic response to metformin: a GoDARTS study. Diabetes. 2009;58: 1434-9.

22. Becker ML, Pearson ER, Tkac I. Pharmacogenetics of oral antidiabetic drugs. Int J Endocrinol. 2013;2013: 686315

23. Pearson ER. Personalized medicine in diabetes: the role of 'omics' and biomarkers. Diabetic Med. 2016;33(6):712-7.

24. Lilly's Law' adds tool for research. Available at: https://www.chicagotribune.com/news/ct-xpm-200909-21-0909200354-story.html. Accessed 10 Oct 2019.

25. Estampador AC, Franks PW. Precision medicine in obesity and type 2 diabetes: the relevance of earlylife exposures. Clin Chem. 2018;64(1):130-141.

26. Ragia G, Petridis I, Tavridou A, et al. Presence of CYP2C9* 3 allele increases risk for hypoglycemia in type 2 diabetic patients treated with sulfonylureas. Pharmacogenomics. 2009;10(11):1781-7.

27. Dujic $\mathrm{T}$, Zhou K, Donnelly LA, et al. Interaction between variants in the CYP2C9 and POR genes and the risk of sulfonylurea-induced hypoglycaemia: A GoDARTS Study. Diabetes Obes Metab. 2018;20(1): 211-4. 